\title{
Facilitation of Fear Extinction by D-Cycloserine: Theoretical and Clinical Implications
}

\author{
Rick Richardson, ${ }^{1}$ Lana Ledgerwood, and Jacquelyn Cranney ${ }^{1}$ \\ School of Psychology, University of New South Wales, Sydney, New South Wales 2052, Australia
}

\begin{abstract}
Anxiety disorders are among the most common psychological disturbances in the industrialized world. Current behavioral therapy procedures for these disorders are somewhat effective, but their efficacy could be substantially improved. Because these procedures are largely based on the process of extinction, manipulations that enhance extinction may lead to improvements in treatment effectiveness. We review the evidence that D-cycloserine (DCS), a partial NMDA agonist, facilitates extinction of learned fear in rats. Although only a few studies have examined the effects of DCS on extinction of learned fear, this work suggests that this drug may have a number of potential clinical benefits. In addition, attempts at interpreting this research illustrate our limited understanding of the processes involved in extinction.
\end{abstract}

A substantial percentage of the industrialized world's population will suffer from an anxiety disorder at some point, and consequently, the burden-of-disease costs (both financial and personal) associated with these disorders are extremely high (e.g., Andrews et al. 2001). As a result, there is considerable interest in developing effective treatments for anxiety disorders. Many current therapies for anxiety disorders (e.g., systematic desensitization, flooding, and implosion) involve controlled exposures to the feared stimulus. In other words, these therapies are based on the process of extinction. Although these therapies are the most effective current form of treatment for anxiety disorders (Foa 2000), they could be improved in a number of ways. For example, some patients who begin treatment fail to complete it (i.e., they drop out after a few sessions), and some patients who successfully complete treatment later relapse (i.e., their symptoms return). Manipulations that enhance the process of extinction might reasonably be expected to lead to an improved efficacy of these therapies. Specifically, if such manipulations reduce the number of sessions required, then fewer patients may fail to complete treatment. If these manipulations also somehow reduced the incidence of relapse, then their benefits would be even greater.

In the past decade, considerable effort has been directed at increasing our understanding of extinction. Much of this research has involved Pavlovian conditioned fear. In such studies, an animal is first exposed to pairings of an initially neutral conditioned stimulus (CS; e.g., light or tone) with a naturally aversive unconditioned stimulus (US; e.g., shock). After this experience, presentations of the CS elicit a number of behaviors usually associated with the state of fear (e.g., tachycardia, elevated blood pressure, potentiated startle response). These learned fear responses can be reduced, however, by repeatedly presenting the CS by itself; in other words the learned fear responses can be extinguished (see Myers and Davis 2002, for operational and conceptual definitions of extinction). Although extinction is a very simple process procedurally, it has proven to be quite complex theoretically.

\section{Theories of Extinction}

A number of theoretical accounts have been offered for extinction. A detailed description of the various theories of extinction

\footnotetext{
1 Corresponding authors.

E-MAIL r.richardson@unsw.edu.au; FAX (61) 293853641.

E-MAIL j.cranney@unsw.edu.au; FAX (61) 293853641.

Article and publication are at http://www.learnmem.org/cgi/doi/10.1101/ Im.78204.
}

is beyond the scope of the present paper, but even a brief overview highlights the fact that very different underlying mechanisms have been proposed in the various theoretical accounts. For example, some have suggested that extinction is due to the "unlearning" of the CS-US association (e.g., Rescorla and Wagner 1972). Others have suggested that extinction is due to a devaluation of the US representation (e.g., Rescorla 1973), or to the learning of a competing association (i.e., CS now predicts "no US") that interferes with the expression of the original CS-US association (e.g., Bouton 1991). From both of these latter perspectives, the original CS-US association stays intact following extinction. Therefore, these theoretical approaches, in contrast to the unlearning approach, can account for the common finding that fear often returns following extinction. For example, presentation of an extinguished CS in a context different from that where the extinction trials occurred often leads to a return of learned fear responses, a phenomenon usually referred to as "renewal" (e.g., Bouton and Bolles 1979a). In addition, presentation of the aversive US, or some other stressful experience, can lead to the recovery of extinguished fear responses, a phenomenon usually referred to as "reinstatement" (e.g., Bouton and Bolles 1979b). If extinction caused the "unlearning" of the CS-US association, then it is difficult to explain either of these effects. In contrast, if extinction is caused by a devaluation of the US representation, and the CS elicits this devalued representation at test, then re-exposure to the US prior to test would restore its representation and lead to the reinstatement of the learned fear responses. If extinction involves the learning of a new inhibitory association (i.e., CS-no US), and that new learning is contextmediated, as is commonly assumed (e.g., Bouton 1993), then testing animals in a context different from that used for extinction would lead to the renewal of learned fear responses (i.e., the original, still intact, CS-US association would be expressed). However, it is important to note that renewal and reinstatement rarely result in learned fear responses being returned to preextinction levels. That is, extinction might involve unlearning as well as these other processes. Therefore, it may be necessary to develop hybrid models of extinction that explicitly allow for the involvement of multiple processes (see Weidemann and Kehoe 2003, for examples of hybrid models of extinction).

\section{Neural Bases of Extinction}

Considerable recent attention has been focused on the neural bases of extinction, and there are several excellent recent reviews 
of this work (e.g., Myers and Davis 2002; Delamater 2004). In brief, some researchers have focused on the role of various brain regions in extinction, but as noted by Myers and Davis (2002), the search for definitive structural sites where extinction occurs has not yet been successful. For example, there is some striking recent evidence that the prefrontal cortex (PFC) is involved in extinction (Milad and Quirk 2002), but others have failed to find evidence that the PFC is involved in extinction (e.g., Gewirtz et al. 1997). In contrast to this structural approach, other researchers have focused on the role of various neurotransmitter systems in extinction. Here there seems to be incontrovertible evidence that NMDA is critically involved in extinction. The vast majority of this evidence involves demonstrations that NMDA antagonists block extinction (for recent review, see Myers and Davis 2002). For example, Falls et al. (1992) reported that infusion of AP5 into the basolateral nucleus of the amygdala (BLA) prior to extinction training dose-dependently blocked extinction of conditioned fear, as measured by fear-potentiated startle. Studies using alternative NMDA antagonists and alternative fear responses have also provided evidence for the involvement of the NMDA system in extinction (Cox and Westbrook 1994; Santini et al. 2001). In addition to demonstrations that NMDA antagonists block extinction, it was very recently found that an NMDA agonist can $\mathrm{fa}$ cilitate extinction of learned fear. Given the potential clinical significance of this latter finding, the present paper reviews that evidence in detail.

\section{Empirical Evidence that NMDA Agonists Facilitate Extinction}

Walker et al. (2002) reported the first evidence that an NMDA agonist facilitates extinction of learned fear. Those investigators used D-4-amino-3-isoxazolidone (D-cycloserine, DCS), a partial agonist that acts at the strychnine-insensitive glycinerecognition site of the NMDA receptor complex, in their study. Unlike many NMDA agonists, DCS does not appear to lead to excitotoxicity (Wlaz et al. 1994; Baran et al. 1995). Walker et al. (2002) found that either systemic administration or BLA infusions of DCS prior to extinction training enhanced the extinction of learned fear in rats, as measured by fear-potentiated startle. Basically, adult rats were first given 10 pairings of a light CS with a shock US. Two days later, some rats were given 30 extinction trials with the light CS (i.e., no US presented on these trials). Shortly prior (30 $\mathrm{min})$ to these extinction trials, rats were injected with DCS or saline. The following day, all rats were tested for fear of the light CS. Specifically, the amplitude of the acoustic startle response was measured at test; on some test trials the acoustic startle stimulus was presented by itself, and on other trials it was preceded by the light CS. If the light elicits fear, then startle amplitude will be greater on those trials where the light CS is presented. The results showed that rats given DCS prior to the extinction trials exhibited less fear of the light CS at test than did the rats given saline (i.e., the DCS facilitated extinction of learned fear). This effect of DCS was only observed in rats given the extinction trials (i.e., the lower level of fear observed in the DCS-treated rats was not due to some nonspecific drug effect). Further, the effect of DCS on extinction was dose-dependent such that rats systemically injected with either $15 \mathrm{or} 30 \mathrm{mg} / \mathrm{kg}$ of DCS exhibited facilitated extinction of learned fear (relative to saline-treated rats), but rats given $3.25 \mathrm{mg} / \mathrm{kg}$ of DCS responded similarly to the saline-treated controls.

These findings were the first to demonstrate that an NMDA agonist facilitates extinction, and they complement earlier work that had established that NMDA antagonists block extinction. Perhaps even more importantly, these findings may have direct clinical significance, because DCS has been approved for use in humans (to treat tuberculosis) and therefore would be relatively safe to use with patients suffering from anxiety disorders. Despite the robustness of the effects reported by Walker et al. (2002), it is only one study. Therefore, we recently started examining the effects of DCS on extinction of learned fear in rats in order to confirm the initial findings of Walker et al. (2002) and to begin to explore the mechanism by which DCS facilitates extinction. The results of our experiments clearly confirm that DCS facilitates extinction of learned fear. In addition, our findings suggest that DCS may have a number of additional effects on extinction, some of which have potential clinical significance.

\section{Effects of Postextinction Administration of DCS}

Ledgerwood et al. (2003) examined the effects of DCS on extinction of learned fear in an attempt to replicate, and extend, the findings reported by Walker et al. (2002). In the study by Ledgerwood et al. (2003), rats were first given five pairings of a 10-sec light CS with a 0.8-mA, 0.8-sec shock US. On the following day, some rats were given six nonreinforced presentations of the light CS (each 2 min long). Immediately after these extinction trials, half the rats were injected with DCS and the others were injected with saline. Other rats were injected with either DCS or saline on this day, but not given the extinction trials. At test, on the day after the extinction session, the light CS was presented for $2 \mathrm{~min}$, and the level of freezing, a species-specific defense response often used to measure fear (Fanselow 1980), was recorded. It was found that rats given DCS after the extinction trials exhibited less fear of the light CS at test than the rats given saline after the extinction trials or the rats injected with DCS but not given any extinction trials.

These results support those reported by Walker et al. (2002), and also show that DCS is effective at facilitating extinction when given after the extinction trials, rather than before. We interpreted these findings as evidence that DCS facilitated the consolidation of a new memory acquired during extinction (i.e., the CS predicts no US). Others have suggested that the NMDA system is involved in the consolidation of extinction memories (e.g., Santini et al. 2001). This idea that DCS facilitated memory consolidation was further supported by our subsequent finding that increasing the delay between the end of extinction and DCS administration leads to a linear decrease in the enhancement effect (Fig. 1; Ledgerwood et al. 2003), with significant facilitation of extinction occurring only when the DCS was administered less than $4 \mathrm{~h}$ after the extinction trials. We, like Walker et

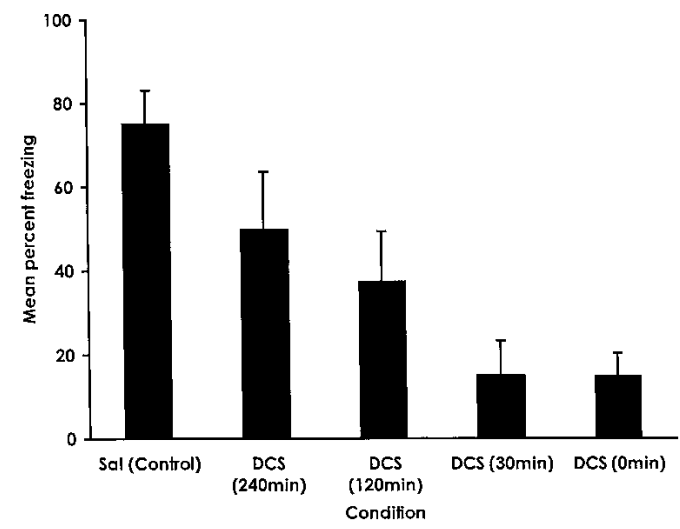

Figure 1 Effect of varying the delay of DCS administration following extinction training. Mean (+SEM) percentage of time rats spent freezing during one 2-min presentation of the light conditioned stimulus (CS) during an extinction retention test. Saline or DCS was administered 240 min, $120 \mathrm{~min}, 30 \mathrm{~min}$, or immediately after extinction training. From Ledgerwood et al. (2003), Experiment 4. 
al. (2002), also found that the facilitation of extinction by a systemic injection of DCS was dose-dependent (optimal dosage $=15$ $\mathrm{mg} / \mathrm{kg}$ ), and that direct infusion of DCS into the BLA, an NMDAreceptor-rich area, was effective at facilitating extinction.

Therefore, we replicated the Walker et al. (2002) initial finding that DCS facilitates extinction of learned fear. In addition, we extended that result to a different measure of fear (i.e., freezing rather than potentiated startle), and also showed that DCS was effective at facilitating extinction of learned fear even when it was given shortly after (rather than before) the extinction trials. The simplest explanation for these findings is in terms of the currently dominant view of extinction: extinction involves new learning (i.e., CS-no US), and DCS facilitates either the acquisition or the consolidation of this new learning by stimulating NMDA receptors in the BLA.

After this initial study, we began to explore in more detail how DCS facilitates extinction. Although this work has not yet definitely determined the mechanism(s) by which DCS facilitates extinction of learned fear, it has shown that DCS may have some additional beneficial clinical effects.

\section{Does DCS Cause “Generalized” Extinction?}

In one study (L. Ledgerwood, R. Richardson, and J. Cranney, in prep.), we examined several issues concerning the DCSenhancement of extinction. First, we examined whether this effect occurs when an aversive US other than shock was used. Second, we compared reacquisition of learned fear responses to an extinguished CS in rats given DCS or saline after extinction (the saline-treated rats had been given additional extinction trials in order to equate levels of fear prior to the reconditioning session). The idea here was to determine whether DCS-treated rats were somehow permanently less fearful of aversive CSs. Third, and most interesting, we examined whether rats given DCS after extinction trials with one CS responded any differently to a second, nonextinguished CS (i.e., do DCS-treated rats exhibit generalized extinction?).

In the first experiment of that study, rats were given 14 pairings of a 20 -sec light CS with a $120-\mathrm{dB}, 100-\mathrm{msec}$ loud noise US. On the following day, rats were given six nonreinforced exposures (each $2 \mathrm{~min}$ in duration) to the light CS (i.e., extinction), and then systemically injected with either DCS $(15 \mathrm{mg} / \mathrm{kg})$ or saline. At test, the day after the extinction session, the DCStreated rats exhibited less freezing to the light CS than did the saline-treated rats. In other words, DCS once again facilitated extinction of learned fear, and in this case the aversive US was a loud noise rather than shock. Giving the saline-treated rats an extra day of extinction trials, however, equated their performance to that observed in the DCS-treated rats. The results of this experiment also demonstrated that DCS-treated rats were similar to saline-treated rats (that had been given an additional extinction session) in subsequently re-acquiring fear of the CS (in a drug-free state). In other words, the rats given DCS following extinction were not permanently impaired in learning to fear a CS.

In the second experiment of that study, rats were initially trained with two CSs (i.e., a light and a tone); each of these CSs was paired with the loud noise US 14 times. On the following day, some rats were given two sessions (separated by $2 \mathrm{~h}$ ) of extinction training with the light CS. Each of these extinction sessions consisted of six nonreinforced exposures to the light CS (each 2 min in duration). Immediately following the second extinction session, some rats were injected with DCS $(15 \mathrm{mg} / \mathrm{kg})$ and others were injected with saline. Other rats in this experiment were injected with saline or DCS on the same day, but not given the extinction trials with the light CS. At test, on the day after the extinction sessions, the light and the tone CS were each presented separately (test order was counterbalanced), and the level of fear, as measured by freezing, was assessed. Performance to the light CS replicated earlier findings (Fig. 2A). That is, rats given DCS following extinction trials exhibited less fear of the light CS than rats given saline after extinction and those injected with DCS but not given the extinction trials with the light CS. The most interesting results of this experiment came from the test with the tone CS, which had not been extinguished for any of the rats. It was found that those rats given the light CS extinction trials and injected with DCS exhibited reduced fear of the tone CS as well (Fig. 2B). That is, the DCS-treated rats exhibited generalized extinction of fear. This effect was not observed in rats injected with saline after the light CS extinction trials or in rats injected with DCS but not extinguished to the light CS. Taken together, the results of this experiment show that DCS facilitated extinction to the nonreinforced CS (i.e., the light) and also reduced fear of a nonextinguished CS as well. This suggests that DCS may have multiple beneficial effects in clinical settings.

One interpretation of this finding of generalized extinction following DCS administration is that DCS facilitates extinction by enhancing the devaluation of the US representation. The notion of US devaluation is best illustrated by a study by Rescorla (1973) in which rats received pairings of a CS with a loud noise US. After this, some rats were habituated to the loud noise US. Those rats habituated to the noise US exhibited less fear of the CS (measured by lick suppression) than those rats not habituated to the noise US. In a subsequent study, Rescorla and Heth (1975) suggested that extinction following nonreinforced presentations of the CS could result from the same process. That is, the CS elicits a representation of the US during the extinction trials. However, given that no US is presented, the animal devalues the US representation. After several such trials, the CS elicits a devalued US representation; one that is incapable of eliciting learned fear responses. In other words, the CS-US association is still intact, but now the CS activates a US representation that is too weak to elicit fear responses.

In the Ledgerwood et al. study (L. Ledgerwood, R. Richardson, and J. Cranney, in prep.; Experiment 3) we extended Rescorla's original finding that US habituation reduced learned fear responses to a CS previously paired with that US. That is, in our experiment rats were given separate pairings of a light CS and a tone CS with a loud noise US. Some rats were then habituated to the noise US. The rats habituated to the noise US exhibited much less freezing to both CSs than did rats not habituated to the noise

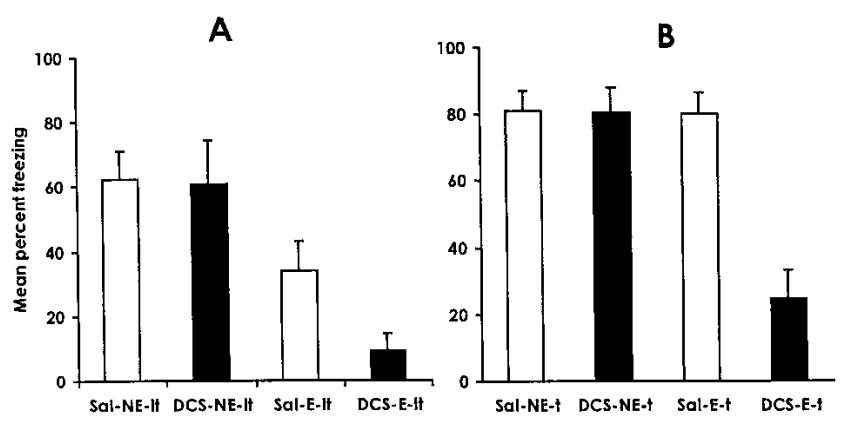

Figure 2 Effect of D-Cycloserine (DCS) on conditioned freezing during test. $(A)$ Mean (+SEM) percent of time rats spent freezing during presentations of the light CS following either extinction training to the light, or handling. Sal, saline; NE, no extinction; $E$, extinction; It, light CS. (B) Mean (+SEM) percent of time rats spent freezing during presentations of the tone CS following either extinction training to the light, or handling. Sal, saline; NE, no extinction; $E$, extinction; $t$, tone CS. From L. Ledgerwood, R. Richardson, and J. Cranney (in prep.), Experiment 2. 
US. In other words, the rats habituated to the US prior to test exhibited a pattern of performance just like that seen in rats given DCS following light CS extinction trials.

Taken together, the results of the study by Ledgerwood et al. (L. Ledgerwood, R. Richardson, and J. Cranney, in prep.) replicate the finding that DCS facilitates extinction of learned fear, and show that these rats are not impaired in re-acquiring fear of the CS. In addition, the results suggest that DCS-treated rats generalize extinction to other CSs previously paired with the US. Indeed, the DCS-treated rats exhibit a pattern of performance at test that is exactly like that seen in rats that are habituated to the US prior to test. Therefore, one might be tempted to conclude that DCS facilitates extinction of learned fear by somehow affecting the devaluation of the US representation associated with the CS(s). Specifically, DCS may not only enhance the rate at which the US representation is devalued (which leads to a faster rate of extinction), but it may also reduce the CS-specificity of this process. That is, extinction trials with one CS rarely lead to a loss in responding to a different CS previously paired with that US (e.g., Richards and Sargent 1983; also see the results of our salinetreated rats in Fig. 2). Therefore, CS extinction trials must not lead to a general devaluation of the US representation (i.e., like that seen with US habituation), but, rather, a stimulus-gated devalued US representation. It appears, however, that injecting DCS after CS extinction trials may lead to a more general devaluation of the US representation.

The generality of this effect remains to be determined. For example, does it occur when a different US is paired with each CS? In our study, both CSs had been paired with a common US (a loud noise). Perhaps the best way to test the generality of this finding, and to gain additional information about the effects of DCS on extinction, would be to first determine whether this drug facilitates extinction of an appetitive CS. To our knowledge, only one study has examined the effects of DCS on the extinction of an appetitive CS. Specifically, Port and Seybold (1998) demonstrated that pre-extinction training DCS administration prevented the extinction of an appetitive bar-press response, whereas preextinction training administration of MK-801 (an NMDA antagonist) enhanced extinction (a pattern opposite to that seen in studies of learned fear). However, the findings reported by Port and Seybold (1998) relate to the extinction training session only (i.e., when the animals were in the drug state), and could therefore be due to the effects of these agents on appetitive motivation. Further work is clearly needed in this area. Such research would not only determine the generality of the DCS enhancement of extinction effect, but also provide a perfect situation for testing the limitations of the generalized extinction effect described above. That is, in this case, rats could be given pairings of CS1-food and CS2-shock. One CS could then be extinguished and the rats injected with either saline or DCS. If DCS facilitates extinction of both appetitive and aversive associations, then the rats should respond less to the extinguished CS when given DCS than when given saline. However, because these USs have different sensory and affective properties (cf. Wagner and Brandon 1989), one might not observe any generalized extinction (i.e., responding to the nonextinguished CS should be the same in these rats as in rats not given extinction trials with either CS).

\section{Reinstatement of Learned Fear in DCS-Treated Rats}

As noted earlier, there are numerous demonstrations that reexposing rats to the US prior to test leads to a recovery of learned fear responses. One interpretation of this finding is that extinction leads to a devaluation of the US representation, and representing the US then restores its representation to its original level (e.g., Rescorla and Heth 1975). If DCS facilitates extinction by enhancing the devaluation of the US representation, then re-presenting the US prior to test should lead to a recovery in learned fear responses. That is, DCS-treated rats should exhibit a reinstatement effect. We examined this prediction in a recent study (Ledgerwood et al. 2004). In that study, rats were first given five pairings of a 10-sec light CS with a $0.8-\mathrm{mA}, 0.8$-sec shock US. On the following day, rats were given six extinction trials with the light CS (each 2 min in duration) and then administered either DCS $(15 \mathrm{mg} / \mathrm{kg})$ or saline. In order to equate levels of fear prior to reinstatement, the saline-treated rats were given an additional day of extinction trials. Some rats were then re-exposed to the shock US $24 \mathrm{~h}$ prior to test. The results showed that rats in the saline condition exhibited reinstatement of fear following the US re-exposure, but the DCS-treated rats failed to do so. A second experiment in that study replicated our initial finding, and also demonstrated that the saline-treated rats only exhibited reinstatement if the pre-test US was given in the same context as that used for extinction and test. Saline-treated rats re-exposed to the shock US in a context different from that used for extinction and test did not exhibit any reinstatement (see Bouton and Bolles $1979 \mathrm{~b}$ for another demonstration of the context-specificity of the US reinstatement effect). The DCS-treated rats in this second experiment failed to exhibit reinstatement regardless of where the US was presented (Fig. 3). In contrast to the Ledgerwood and colleagues' findings described above (L. Ledgerwood, R. Richardson, and J. Cranney, in prep.), these results do not provide support for the hypothesis that DCS facilitates extinction by devaluing the US representation. Nevertheless, they do suggest that this drug may have clinical benefits in addition to speeding up the rate of extinction. Specifically, it may be the case that DCStreated patients may be less likely to relapse following successful completion of treatment.

As noted, these results raise questions about the hypothesis that DCS facilitates extinction by enhancing the devaluation of the US representation. Because DCS-treated rats failed to exhibit a reinstatement effect, it would appear that DCS enhances extinction through some other process. One possibility is that DCS facilitates extinction by enhancing the learning of an inhibitory association (i.e., CS-no US) that then competes with the original, and still intact, CS-US association for control of conditioned responding. Because DCS is an NMDA agonist, and activation of the NMDA system has been shown to be critically important for various types of learning (Collingridge and Bliss 1987; Lee and Kim 1998), it would be reasonable to assume that DCS facilitates the learning of this inhibitory association. Bouton (1993) suggested that this inhibitory CS-no US association is contextspecific, and that representing the US in the same context as extinction training and test disrupts the occasion-setting prop-

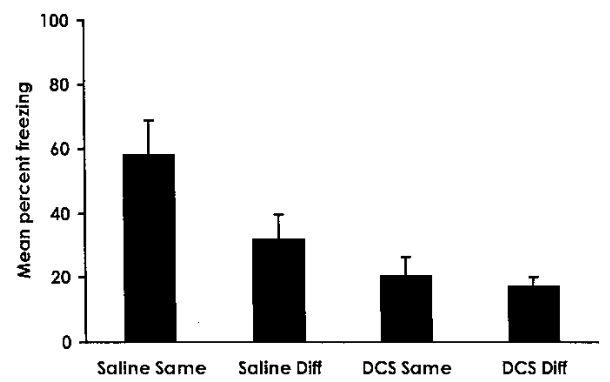

Figure 3 Effect of $D$-Cycloserine (DCS) on conditioned freezing during the extinction retention test following US reinstatement in either the same context or a context different from that of extinction training. Mean (+SEM) percent of time rats spent freezing during four 30-sec presentations of the light CS in Experiment 2. Same, same context as reinstatement; Diff, different context. From Ledgerwood et al. (2004), Experiment 2. 
erties of that context and leads to expression of the original CSUS association. However, the failure to observe reinstatement in the DCS-treated rats would appear to also be problematic for this particular theoretical approach to extinction.

One possible explanation of the failure to see reinstatement in rats given DCS after extinction, while still maintaining a new learning account of extinction, would be that DCS-injected rats develop an extremely strong (and possibly context-independent) inhibitory CS-US association. For example, it has been shown that massive extinction (i.e., 800 extinction trials) reduces renewal of fear (Denniston et al. 2003). If DCS causes the effects of extinction to become context-independent after only a few trials, then reinstatement might not be as straightforward to demonstrate in these rats as it is in saline-treated rats. That is, it may be necessary to provide stronger, or more, US exposures in order to observe reinstatement in the DCS-treated animals. Clearly, further research is required to examine this possibility. Whatever the outcome of these future investigations, however, the finding that rats given DCS after extinction fail to exhibit reinstatement of learned fear in a situation where saline-treated rats, given additional extinction trials in order to equate levels of extinction, do exhibit reinstatement, highlights another potential clinical benefit of this drug. That is, not only does DCS facilitate extinction of learned fear (Walker et al. 2002; Ledgerwood et al. 2003), and appear to produce generalized extinction (L. Ledgerwood, R. Richardson, and J. Cranney, in prep.), it also seems to reduce the occurrence of reinstatement-induced relapse (Ledgerwood et al. 2004). It will be interesting to determine whether rats given DCS after extinction also exhibit less renewal and spontaneous recovery of learned fear responses compared to rats given saline after extinction. Both of these phenomena are clinically relevant, and based on our results with reinstatement, we predict that DCStreated rats will exhibit less renewal and spontaneous recovery than saline-treated rats.

\section{Effects of Repeated Exposures to DCS on Its Effectiveness as a Facilitator of Extinction}

In another series of experiments, we examined whether multiple exposures to DCS affects its ability to facilitate extinction of learned fear (A. Parnas, M. Weber, and R. Richardson, in prep.). In all three experiments in that study, rats were first given five pairings of a 10 -sec light CS with a $0.8-\mathrm{mA}, 0.8$-sec shock US. In the first experiment we simply replicated the results reported by Ledgerwood et al. (2003). That is, rats given DCS $(15 \mathrm{mg} / \mathrm{kg})$ immediately after six extinction trials with the light CS (each 2 min in duration) exhibited less fear at test (i.e., they exhibited less freezing in response to the light CS) than did rats given saline after the extinction trials or rats given DCS but not extinguished. In the second experiment, we examined the effects of 0,1 , and 5 pre-exposures to DCS on its ability to facilitate extinction of learned fear. Specifically, rats in all three conditions were injected once every other day for $10 \mathrm{~d}$. These injections were of either DCS (0, once, or all five times) or saline (on any occasion where DCS was not injected). After this 10-day period, all rats were given five pairings of the light CS with a shock US. On the following day, rats were given six nonreinforced presentations of the light CS (each 2 min in duration) and then injected with DCS. The performance of these three groups was compared to that of a group given saline after the extinction trials. The rats given a postextinction injection of DCS exhibited facilitated extinction, compared to saline-injected controls, if they had not been pre-exposed to DCS (Fig. 4). However, those rats injected with DCS after extinction, but also given five previous injections of DCS (during the preceding $10 \mathrm{~d}$ ), performed just like the rats given saline after extinction. In other words, DCS failed to facili-

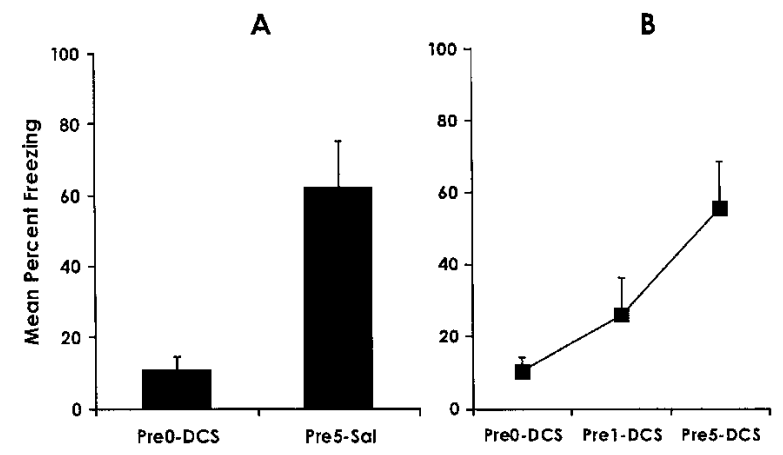

Figure 4 (A) Mean (+ SEM) percentage of freezing to the light CS during the extinction retention test for rats that had received five DCS injections prior to conditioning and a saline injection following extinction training (Pre5-Sal) and rats that received five saline injections prior to conditioning and a DCS injection after extinction training (Pre0-DCS). (B) Mean (+SEM) percentage of freezing to the light CS during the extinction retention test for rats that received either five DCS injections prior to conditioning (Pre5-DCS), one DCS injection prior to conditioning (Pre1DCS), or no DCS injections prior to conditioning (Pre0-DCS). From A. Parnas, M. Weber, R. Richardson (in prep.), Experiment 2.

tate extinction of learned fear in rats that had been previously exposed to DCS. This finding was replicated in the third experiment, which also showed that interpolating a 4-wk interval between DCS pre-exposures and its use in the postextinction period restored its ability to facilitate extinction. That is, giving DCS repeatedly seems to reduce its effectiveness at facilitating extinction, but this effect is not permanent. If a sufficient interval of time is interpolated between DCS exposures and its use as an agent to facilitate extinction, then it is still effective at facilitating extinction of learned fear.

One possible explanation of the failure of DCS to enhance extinction after multiple exposures is that the DCS pre-exposures shifts the dose-response curve to the right (i.e., the development of tolerance). However, a previous study demonstrating desensitization to DCS following chronic exposure to it failed to provide any evidence to support this tolerance account (Quartermain et al. 1994). That is, those authors found that acute treatment with DCS facilitated maze learning in mice. They also found that if the mice were pre-exposed to $3 \mathrm{mg} / \mathrm{kg}$ DCS twice a day for $15 \mathrm{~d}$ prior to training, the enhancing effects of DCS on performance were abolished. Further, after $15 \mathrm{~d}$ of DCS pre-exposure, increasing the dose of DCS administered after training did not reverse the desensitizing effects of pre-exposure. Thus, it may be the case that chronic exposure to DCS abolishes its activity at the NMDA receptor. However, the biomolecular mechanisms underlying the loss of NMDA receptor responsiveness to DCS are not yet known (but see Quartermain et al. 1994 for a discussion of possible mechanisms).

At this point, we cannot specify exactly how much time is necessary between DCS exposures for it to remain effective at facilitating extinction. Clearly, however, both the number of DCS exposures and the interval separating these exposures are factors that need to be carefully considered in both experimental studies and in potential clinical settings where DCS may be used. Our results showing that DCS loses its effectiveness, at least temporarily, in facilitating extinction of learned fear following chronic exposure, also illustrate that it may be important to consider what other drugs patients are taking before using DCS in treatment. This concern is of particular interest in terms of drugs that are commonly prescribed to people with anxiety disorders. For example, long-term treatment with selected antidepressants has been shown to modify the function of the glycine/NMDA receptor complex in mice (Popik et al. 2000). More specifically,

\section{Learning \& Memory}


administration of either imipramine or citalopram for $21 \mathrm{~d}$ reduced the anxiolytic effects of L-701,324, an antagonist at the strychnine-insensitive glycine site of the NMDA receptor. The same desensitising effects on glycine/NMDA receptor function were also found after $8 \mathrm{~d}$ of an electroconvulsive shock procedure. Because these various treatments affect the functional activity of glycine-activated NMDA receptors, it would be of interest to examine whether multiple pre-exposures to antidepressants (or electroconvulsive shock) reduce the DCS enhancement of extinction effect. These issues should obviously be carefully considered in any clinical trials of DCS.

\section{DCS and Treatment of Anxiety Disorders in Humans}

Two recent papers have examined the use of DCS in treating an anxiety disorder. Heresco-Levy et al. (2002) reported the results of a double-blind, cross-over design study with Post-traumatic stress disorder (PTSD) patients. During the DCS phase of the study, patients were given $50 \mathrm{mg} / \mathrm{kg}$ of DCS (in two separate doses of $25 \mathrm{mg} / \mathrm{kg}$ ) every day for $4 \mathrm{wks}$; during the other phase of the study, patients were given a placebo for 4 wks. A 2-wk period separated the DCS and placebo phases. Clinical assessments revealed that symptoms were reduced during the DCS phase, but similar improvements were also noted during the placebo phase. Therefore, the results of that study fail to support the idea that DCS could benefit therapeutic recovery. However, there are a number of potential concerns with the study. First, the patients did not appear to have received any cognitive/behavioral therapy in conjunction with the DCS. That is, the effect of the drug per se on the disorder was examined. Based on the preclinical evidence reviewed above, it is not surprising that the drug itself did not lead to greater improvements (i.e., in order to observe facilitated extinction in the studies described above, the rats had to be reexposed to the fear-eliciting stimulus). Second, the sample size was very small in the clinical study (data were available for only 10 patients in the DCS phase). Third, as noted earlier, chronic exposure to DCS may reduce its efficacy in facilitating extinction. Therefore, giving DCS every day for 4 wks may not be an effective strategy. Indeed, in the other study on using DCS to treat an anxiety disorder in humans (Ressler et al. 2004) it was explicitly suggested that acute, rather than chronic, treatment with DCS may be the most effective way of using this drug to facilitate loss of fear. Fourth, the majority of the patients in the study by Heresco-Levy et al. (2002) were currently being treated with an antidepressant. As noted earlier, chronic treatment with antidepressants can alter activity at the NMDA receptor. For all these reasons, one must be cautious in accepting the null results reported by Heresco-Levy et al. (2002).

The more recent preliminary study with humans and DCS has reported more encouraging results (Ressler et al. 2004). In that study, it was found that DCS (50 and $500 \mathrm{mg}$ ) administered orally prior to each of two sessions of virtual reality therapy produced a faster reduction of acrophobia (abnormal fear of heights) than that seen in patients exposed to virtual heights and administered a placebo. These beneficial effects were observed with both physiological and self-report measures, and in both the virtual environment and the real world. In addition, the beneficial effect of DCS in these patients was long-lasting, with reduced levels of fear observed at a 3-mo follow-up. Although this was only a preliminary study (with data for 17 DCS-treated patients), it does suggest that the preclinical work described above may indeed have clinical significance. In contrast to the study by Heresco-Levy et al. (2002), the subjects in the Ressler et al. study (2004) were given explicit exposure-based therapy in conjunction with the DCS. Further, there was no mention that the subjects were currently taking any other drugs for their disorder.
Finally, the selection of acrophobics may have been a particularly astute choice, because this disorder has a specific eliciting stimulus (i.e., heights), and is modifiable by exposure-based therapies.

\section{Conclusions}

The results reviewed above show that DCS, a partial NMDA agonist, facilitates extinction of learned fear. Although there are some limitations to the DCS enhancement of extinction effect (e.g., the drug temporarily loses its effectiveness with multiple exposures), this finding provides further evidence that NMDA is critically involved in extinction. The finding that an NMDA agonist facilitates extinction of learned fear also has substantial clinical significance, because the most effective current treatment for anxiety disorders (i.e., exposure therapy) is based on extinction. In addition, our work suggests that rats given DCS after extinction exhibit generalized extinction. That is, rats given DCS after one particular aversive CS has been extinguished also exhibit less fear to a second, nonextinguished aversive CS. Further, we have shown that rats given DCS after extinction fail to exhibit reinstatement of learned fear responses in a situation where salinetreated rats do exhibit such an effect. Both of these findings suggest that DCS may have clinical value beyond its ability to enhance the rate at which extinction occurs. Both of these findings also raise some questions about the theoretical process(es) by which extinction occurs. That is, it is difficult to account for these findings with current theoretical models of extinction, and it may be necessary to develop multiprocess theories of extinction in order to fully explain these findings, as well as other results in the extinction literature.

\section{REFERENCES}

Andrews, G., Henderson, S, and Hall, W. 2001. Prevalence, comorbidity, disability, and service utilization: Overview of the Australian National Mental Health Survey. Br. J. Psychiatry 178: 145-153.

Baran, H., Gramer, M., and Loscher, W. 1995. Alterations in plasma and brain amino acids after administration of the glycine/NMDA receptor partial agonist, D-Cycloserine, to mice and rats. Eur. J. Pharmacol. 273: 197-201.

Bouton, M.E. 1991. A contextual analysis of fear extinction. In Handbook of behavior therapy and psychological science: An integrative approach (ed. P.R. Martin), pp. 435-453. Pergamon Press, Elmsford, NY.

. 1993. Context, time, and memory retrieval in the interference paradigms of Pavlovian conditioning. Psychol. Bull. 114: 80-99.

Bouton, M.E. and Bolles, R.C. 1979a. Contextual control of the extinction of conditioned fear. Learn. Motiv. 10: 445-466. 1979b. Role of contextual stimuli in reinstatement of extinguished fear. J. Exp. Psychol. Anim. Behav. Process. 5: 368-378.

Collingridge, G.L. and Bliss, T.V.P. 1987. NMDA receptors-Their role in long-term potentiation. Trends Neurosci. 10: 288-293.

Cox, J. and Westbrook, R.F. 1994. The NMDA receptor antagonist MK-801 blocks acquisition and extinction of conditioned hypoalgesia responses in the rat. Q. J. Exp. Psychol. B 47: 187-210.

Delamater, A.R. 2004. Experimental extinction in Pavlovian conditioning: Behavioural and neuroscience perspectives. Q. J. Exp. Psychol. 57(B): 97-132.

Denniston, J.C., Chang, R.C., and Miller, R.R. 2003. Massive extinction treatment attenuates the renewal effect. Learn. Motiv. 34: 68-86.

Falls, W.A., Miserendino, M.J., and Davis, M. 1992. Extinction of fear-potentiated startle: Blockade by infusion of an NMDA antagonist into the amygdala. J. Neurosci. 12: 854-863.

Fanselow, M.S. 1980. Conditioned and unconditioned components of post-shock freezing. Pavlov. J. Biol. Sci. 15: 177-182.

Foa, E. 2000. Psychosocial treatment of posttraumatic stress disorder. J. Clin. Psychiatry 61: 43-48.

Gewirtz, J.C., Falls, W.A., and Davis, M. 1997. Normal conditioned inhibition and extinction of freezing and fear potentiated startle following electrolytic lesions of medial prefrontal cortex. Behav. Neurosci. 111: 712-726.

Heresco-Levy, U., Kremer, I., Javitt, D.C., Goichman, R., Reshef, A., Blanura, M., and Cohen, T. 2002. Pilot-controlled trial of D-cycloserine for the treatment of post-traumatic stress disorder. Int J. Neuropsychopharmacol. 5: 301-307.

Ledgerwood, L., Richardson, R., and Cranney, J. 2003. Effects of 


\section{Richardson et al.}

D-Cycloserine on extinction of conditioned freezing. Behav. Neurosci. 117: $341-349$.

2004. D-Cycloserine and the facilitation of extinction of conditioned fear: Consequences for reinstatement. Behav. Neurosci. 118: $505-513$.

Lee, H. and Kim, J.J. 1998. Amygdalar NMDA receptors are critical for new fear learning in previously fear-conditioned rats. J. Neurosci. 18: $8444-8454$.

Milad, M.R. and Quirk, G.J. 2002. Neurons in medial prefrontal cortex signal memory for fear extinction. Nature 420: 70-74.

Myers, K.M. and Davis, M. 2002. Behavioral and neural analysis of extinction. Neuron 36: 567-584.

Popik, P., Wrobel, M., and Nowak, G. 2000. Chronic treatment with antidepressants affects glycine/NMDA receptor function: Behavioral evidence. Neuropharmacology 39: 2278-2287.

Port, R. and Seybold, K. 1998. Manipulation of NMDA-receptor activity alters extinction of an instrumental response in rats. Physiol. Behav. 64: 391-393.

Quartermain, D., Mower, J., Rafferty, M.F., Herting, R.L., and Lanthorn T.H. 1994. Acute but not chronic activation of the NMDA-coupled glycine receptor with D-Cycloserine facilitates learning and retention. Eur. J. Pharmacol. 257: 7-12.

Rescorla, R.A. 1973. Effect of US habituation following conditioning. J. Comp. Physiol. Psychol. 82: 137-143.

Rescorla, R.A. and Heth, C.D. 1975. Reinstatement of fear to an extinguished conditioned stimulus. J. Exp. Psychol. Anim. Behav. 1: 88-96.

Rescorla, R.A. and Wagner, A.R. 1972. A theory of Pavlovian conditioning: Variations in the effectiveness of reinforcement and nonreinforcement. In Classical conditioning II: Current research and theory (eds. A.H. Black and W.H. Prokasy), pp. 64-99. Appleton-Century-Croft, New York.

Ressler, K.J., Rothbaum, B.O., Tannebaum, L., Anderson, P., Graap, K., Zimand, E., Hodges, L., and Davis, M. 2004. Cognitive enhancers as adjuncts to psychotherapy: Use of D-Cycloserine in phobics to facilitate extinction of fear. Arch. Gen. Psychiatry (in press).

Richards, R.W. and Sargent, D.M. 1983. The order of presentation of conditioned stimuli during extinction. Anim. Learn. Behav. 11: 229-236.

Santini, E., Muller, R.U., and Quirk, G.J. 2001. Consolidation of extinction learning involves transfer from NMDA-independent to NMDA-dependent memory. J. Neurosci. 21: 9009-9017.

Wagner, A.R. and Brandon, S.E. 1989. Evolution of a structured connectionist model of Pavlovian conditioning (AESOP). In Contemporary learning theories: Pavlovian conditioning and the status of traditional learning theory (eds. S.B. Klein and R.R. Mowrer), pp. 149-189. Erlbaum, Hillsdale, NJ.

Walker, D.L., Ressler, K.J., Lu, K.-T., and Davis, M. 2002. Facilitation of conditioned fear extinction by systemic administration or intra-amygdala infusions of D-Cycloserine as assessed with fear-potentiated startle. J. Neurosci. 22: 2343-2351.

Weidemann, G. and Kehoe, E.J. 2003. Savings in classical conditioning in the rabbit as a function of extended extinction. Learn. Behav. 31: $49-68$.

Wlaz, P., Baran, H., and Loscher, W. 1994. Effect of the glycine/NMDA receptor partial agonist, D-Cycloserine, on seizure threshold and some pharmacodynamic effects of MK-801 in mice. Eur. J. Pharmacol. 257: $217-225$.

\section{Learning \& Memory}




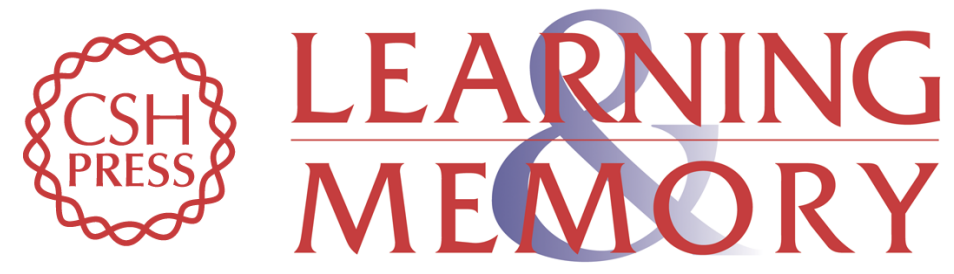

\section{Facilitation of Fear Extinction by D-Cycloserine: Theoretical and} Clinical Implications

Rick Richardson, Lana Ledgerwood and Jacquelyn Cranney

Learn. Mem. 2004, 11:

Access the most recent version at doi:10.1101//m.78204

References This article cites 27 articles, 5 of which can be accessed free at: http://learnmem.cshlp.org/content/11/5/510.full.html\#ref-list-1

License

Email Alerting

Receive free email alerts when new articles cite this article - sign up in the box at the Service top right corner of the article or click here. 